\title{
Real-time Guiding Catheter and Guidewire Detection for Congenital Cardiovascular Interventions
}

\author{
YingLiang $\mathrm{Ma}^{1 *}$, Mazen Alhrishy ${ }^{2}$, Maria Panayiotou ${ }^{2}$, \\ Srinivas Ananth Narayan ${ }^{3}$, Ansab \\ Fazili $^{2}$, Peter Mountney ${ }^{4}$ and Kawal S. Rhode ${ }^{2}$ \\ ${ }^{1}$ School of Computing and Digital Technology, Birmingham City University, UK \\ ${ }^{2}$ Division of Imaging Sciences and Biomedical Engineering, King's College London, UK \\ ${ }^{3}$ Department of Cardiology, Guy's \& St. Thomas' Hospitals NHS Foundation Trust, \\ London, UK \\ ${ }^{4}$ Medical Imaging Technologies, Siemens Healthineers, Princeton, NJ, USA \\ *y.ma@bcu.ac.uk
}

\begin{abstract}
Guiding catheters and guidewires are used extensively in pediatric cardiac catheterization procedures for congenital heart diseases (CHD). Detecting their positions in fluoroscopic X-ray images is important for several clinical applications, such as visibility enhancement for low dose X-ray images, and co-registration between $2 \mathrm{D}$ and $3 \mathrm{D}$ imaging modalities. As guiding catheters are made from thin plastic tubes, they can be deformed by cardiac and breathing motions. Therefore, detection is the essential step before automatic tracking of guiding catheters in live X-ray fluoroscopic images. However, there are several wire-like artifacts existing in X-ray images, which makes developing a real-time robust detection method very challenging. To solve those challenges in real-time, a localized machine learning algorithm is built to distinguish between guiding catheters and artifacts. As the machine learning algorithm is only applied to potential wire-like objects, which are obtained from vessel enhancement filters, the detection method is fast enough to be used in real-time applications. The other challenge is the low contrast between guiding catheters and background, as the majority of X-ray images are low dose. Therefore, the guiding catheter might be detected as a discontinuous curve object, such as a few disconnected line blocks from the vessel enhancement filter. A minimum energy method is developed to trace the whole wire object. Finally, the proposed methods are tested on 1102 images which are from 8 image sequences acquired from 3 clinical cases. Results show an accuracy of $0.87 \pm 0.53 \mathrm{~mm}$ which is measured as the error distances between the detected object and the manually annotated object. The success rate of detection is $83.4 \%$.
\end{abstract}

\section{Introduction}

According to the American Heart Association, Congenital heart diseases (CHD) affect an estimated $0.5 \%$ to $1 \%$ of all live births. They are responsible for up to $40 \%$ of all deaths from congenital anomalies and account for $3.0-7.5 \%$ of all infant deaths. CHD has been traditionally treated with surgery. However, with remarkable advances of interventional cardiology, the majority of congenital heart problems can be treated using cardiac intervention procedures. The procedures are currently guided using X- 
ray fluoroscopy, and generally involve guiding catheters and guidewires, which can be then used to deploy interventional devices. Real-time guiding catheter and guidewire detection, is essential for many image guided applications, such as visibility enhancement for low dose X-ray images, and co-registration between 2D and 3D imaging modalities. As intervention procedures are mainly for young patients with $\mathrm{CHD}$, radiographer has to use the minimum amount of $\mathrm{X}$-ray radiation to guide the procedure. Therefore, the majority of X-ray fluoroscopic images are low dose screening images, with little contrast between guidewires and background. Low dose screening images are of low quality, and are generally acquired to aid navigation (For higher quality, high dose cine images can be acquired, which are well contrasted and less noisy than low dose screening images). Furthermore, guiding catheters are often used to give essential backup support, to assist in pushing interventional equipment over guidewires to the area to be treated. However, the guiding catheters for congenital cardiovascular interventions (CCI) is often made from plastic materials, which are less likely to injure vessels or other structures inside the vascular compartment. However, as these materials are not radiodense, there will be very little contrast for guiding catheters on the X-ray image when compared to the guidewires. Those two challenges have made the detection of catheters more difficult.

Conventional methods using region features, such as pixel intensity, texture and histogram, cannot track guiding catheters or guidewires well [1]. Another category of detection methods, such as active contours and level sets [2,3], can be easily distracted by image artifacts and other wire-like objects (see fig. 1). Ma et al. [12] have developed a catheter detection method based on blob detection. However, this method only works on catheters with electrodes and will not detect guiding catheters or wires. Beyar et al. [4] designed a guidewire detection method by using combination of a filter based method, and the Hough transform, to extract wire-like objects and fit them with polynomial curves. This method would likely fail in our X-ray images, as there is no classification between image artifacts and real wire-like object. Similarly, Baert et al. [5], used image subtraction and template matching to enhance guidewires, but only detected a part of the guidewire. More recently, Barbu et al. [6], Wang et al. [7], and Navab et al. [8], had developed learning-based methods for guidewire tracking. Based on a database of manual annotations for guidewires, Barbu et al., used a marginal space learning method to track the target object. But overall, they only achieved tracking speed of one frame-per-second (fps). Wang et al., utilized a probabilistic framework, and tracking speed of 2 fps was achieved. All tracking methods need manual or semi-automatic initialization of guidewire models. Navab et al. applied a machine learning approach on random generated deformable models to extract guidewires. However, this was not tested on cases where guidewires have a sudden and large deformation movement. 


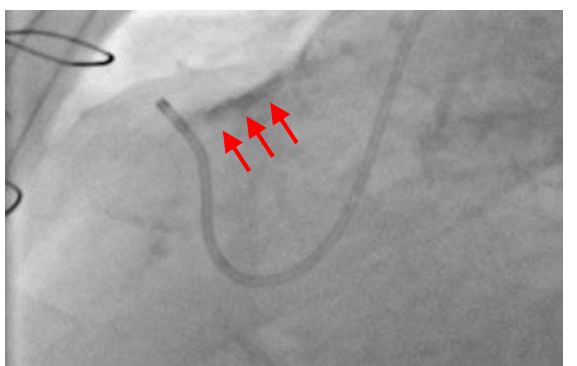

(a) Calcium deposits

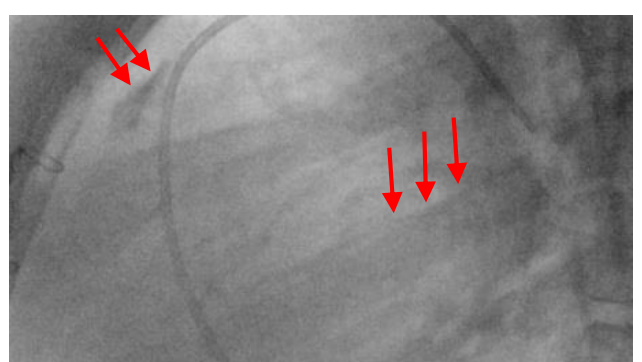

(b) Calcium deposits and rib bones

Fig. 1. Image artifacts (indicated by red arrows) in X-ray images.

To achieve real-time speed, and to overcome challenges of low contrast and image artifacts, in this paper, a localized machine learning algorithm is developed. It is used to classify wire-like objects into two categories: wire objects, and image artifacts. As guiding catheters or guidewires have low contrast against the background in X-ray images, they could be represented as several disconnected wire objects. Therefore, an energy minimum algorithm is developed to find an optimal wire object.

\section{Methods}

During CCIs, the first step is to push a guiding catheter toward the area to be treated, which sometimes involves pushing the catheter into a narrow blood vessel (fig. 2(a)). Then, a guidewire is pushed through the guiding catheter (fig. 2(b)). Finally, a treatment device, such as a balloon or stent, is push along guidewire inside the guide catheter to the target area and deployed.

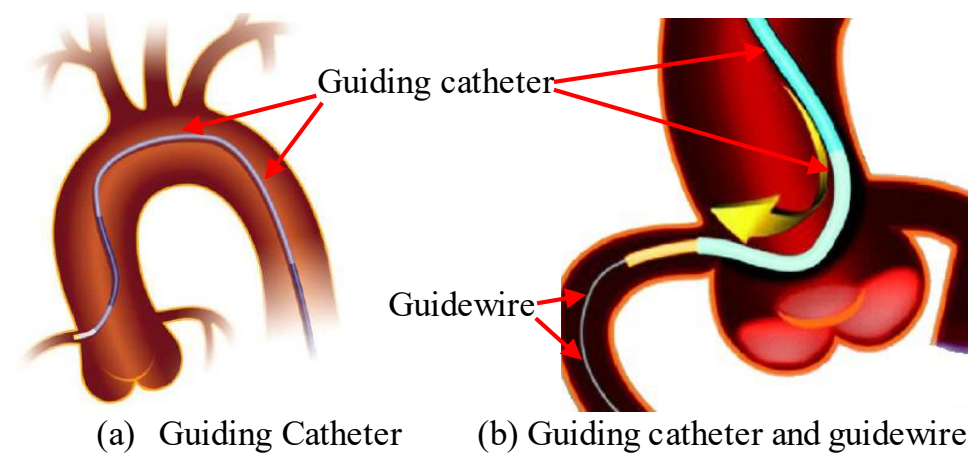

Fig. 2. The Clinical workflow for guiding catheter and guidewire.

Guidewires used in CCIs are different from the guidewires shown in [7], as they have to support interventional devices. Therefore, guidewires are thicker and less flexible than examples shown in [7]. This makes the energy minimum method suitable for finding both guiding catheters and guidewires in CCIs. 


\subsection{Image Pre-processing}

A Multiscale vessel enhancement filter [9] is used to enhance the visibility of wirelike structures in the X-ray images. It is based on the idea of approximating wire-like objects, such as tubular or cylindrical structures [9]. In order to classify wire-likes structures, the vessel filter algorithm finds the local coordinate system aligned with the wire and use the curvature in these directions ( $\mathrm{x}$ or $\mathrm{y}$ axis) to classify different structures. This involves the following 5 steps. Step 1), the algorithm performs Gaussian smoothing by convolving the 2D input image with a Gaussian kernel of the appropriate scale $s$. The Gaussian kernel at position $X=(x, y)$, and scale $s$ is defined as follows:

$$
G(X, s)=\frac{1}{2 \pi s^{2}} \exp \left(-\frac{\|X\|^{2}}{2 s^{2}}\right)
$$

The smoothed image $L(X, s)$ is computed as $L(X, s)=L(X) * G(X, s)$, where $*$ is the convolution operator. Step 2$)$, the algorithm finds an orthogonal coordinate system aligned with the local features in the image by forming and decomposing the $2 \times 2$ Hessian matrix at every image pixel. The Hessian matrix $H_{X, S}$ consists of second order derivatives that contain information about the local curvature. $H_{X, S}$ is defined such as:

$$
H_{X, s}=\left|\begin{array}{ll}
L_{x x}(X, s) & L_{x y}(X, s) \\
L_{y x}(X, s) & L_{y y}(X, s)
\end{array}\right|
$$

Where, $L_{x y}(X, s)=\frac{\partial}{\partial x}\left(\frac{\partial}{\partial y} L(X, s)\right)$, and the other terms are defined similarly. Step 3), the algorithm performs eigenvalue decomposition for Hessian matrix $H_{X, s}$. Step 4), the algorithm uses the eigenvalues and computes the vessel classification. As $H_{X, S}$ is a $2 \times 2$ matrix, there are two eigenvectors and eigenvalues at every image pixel. To quantify any local structures in the image, the eigenvalues for each pixel are arranged in increasing order such that $\left\|\lambda_{1}\right\|<\left\|\lambda_{2}\right\|$. The ratio differentiates between wire-like structures and blob-like structures, and is given by $R=\lambda_{1} / \lambda_{2}$. If $R \approx 1$, detected structures will be blob-like structures. Otherwise, they will be wire-like structures. Step 5), is to apply the vessel filter repeatedly using different Gaussian scales to take into account different vessel sizes within the $2 \mathrm{D}$ image.

The multiscale parameter $s$ is one of the important parameters in the vessel enhancement filter. If $s$ is set too high, guiding wires or catheters will be filtered out. If the range of $s$ is set too large, it will slow down the vessel enhancement filter dramatically. In order to get the optimal result for enhancing guiding catheters, the multiscale $s$ of Gaussian kernel should be centred at the average radius of target objects [9]. To calculate the average radius, radii of several guiding catheters and guidewires used in clinical cases are measured. To convert them into image pixel space, $R_{\text {dicom }}$ pixel to $\mathrm{mm}$ ratio are obtained from X-ray Dicom image header. Magnification factor $M$ of X-ray system is also estimated, which is based on $M=$ $D_{\text {det }} / D_{\text {pat }}$ ( $D_{\text {det }}$ is the distance from X-ray source to the detector, and $D_{\text {pat }}$ is the distance from X-ray source to the patient). The real pixel to $\mathrm{mm}$ ratio is defined as: $R_{\text {xray }}=R_{\text {dicom }} / M$. The final multiscale $s$ is in the range of $2 \leq s \leq 6$ (measured in image pixels). 


\subsection{Object Extraction}

In order to reduce computational complexity, and achieve real-time detection speed, the image, after applying the vessel enhancement filter, is binarized using Otsu's method [10] with an image mask. It is well known that X-ray image formation is governed by Beer-Lambert law [13]. So the contrast of an object depends on the background. In order to minimize the contrast variation, an image mask which covers the target objects (guidewires or guiding catheters) is used to remove the background from the calculation of Otsu's method so that the contrast of target objects is directly related to their radio density. The image mask is created from an image after applying the vessel enhancement filter, which involves downsampling, dilation and thresholding using the average intensity. Figure 3 gives an example. Final step of binarization is to use Otsu' method to calculate the thresholding level only within the area of the image mask. Otsu' method is a non-parametrized and adaptive algorithm as it automatically determines the thresholding level based on minimizing the intraclass variance. Otsu' method has been used together with vessel enhancement filter for coronary sinus segmentation on X-ray image [11].

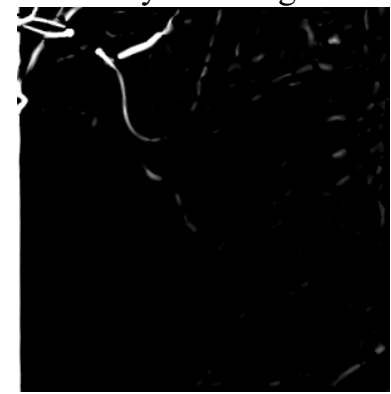

(a)

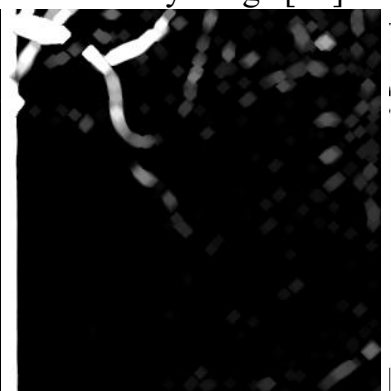

(b)

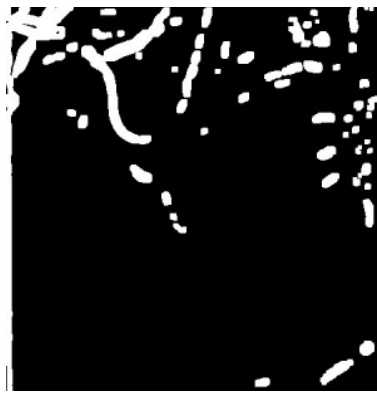

(c)

Fig. 3. (a) Image after applying the vessel enhancement filter. (b) After downsampling and dilation. (c) After thresholding using the average intensity. It is the image mask used by Otsu' method.

After binarization, a standard contour detection algorithm is applied to the binarized image to get contours of wire-like objects. Because wire-like objects, such as guiding catheters or guidewires, have different levels of contrast in X-ray images, they might be detected as a completed contour (fig. 4a), or as several blocks of contours (fig. 4b). Therefore, centerlines need to be computed among blocks of contours as the final results of detection method.

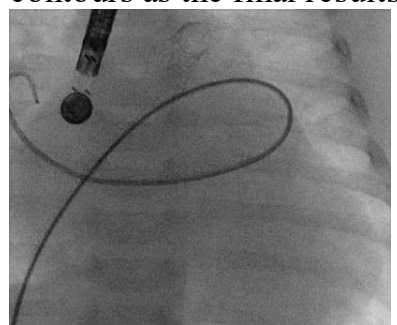

(a)

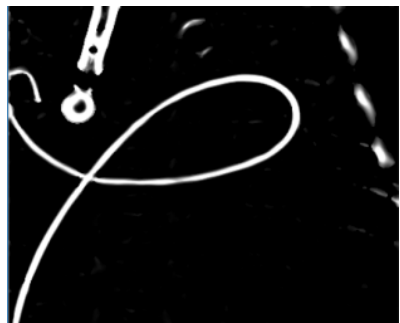

(b)

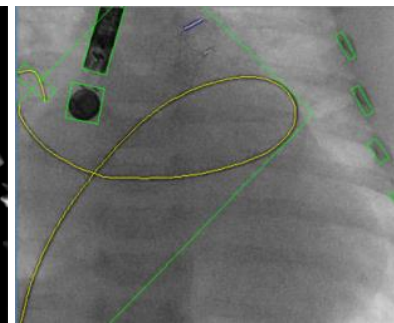

(c) 


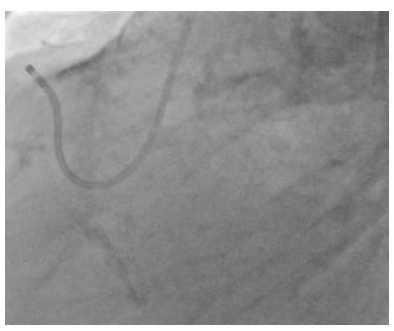

(d)

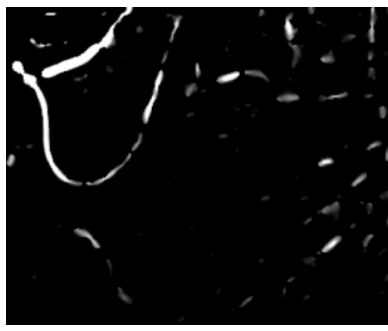

(e)

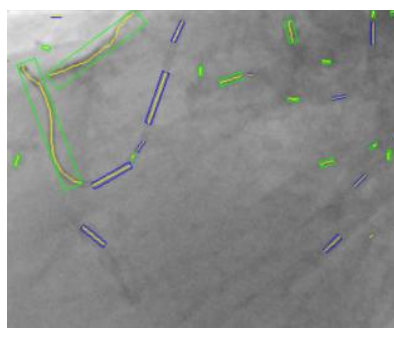

(f)

Fig. 4. (a)(d) Origin X-ray images. (b)(e) Images after applying the vessel enhancement filter. (c)(f) Centerlines of contour blocks. Blue rectangles are the line blocks. The green rectangles are the minimum area rectangles of contour blocks. The yellow curves/lines are centerlines.

To save computational cost, minimum area rectangles of contours are computed. A contour block can be classified as a line block if the width of its minimum area rectangle is less than 8 pixels. 8 pixels is calculated from the average of multiscale $\bar{s}=\frac{2+6}{2}=4$, and $8=2 \bar{s}$ as the width of minimum area rectangle should be the diameter of wire-like object. If a contour block is a line block, the centerline can be approximated by the middle straight line of the minimum area rectangle (fig. $4 \mathrm{f}$ ). Otherwise, the centerline of the contour block can be computed by using the algorithm described in fig. 5 .

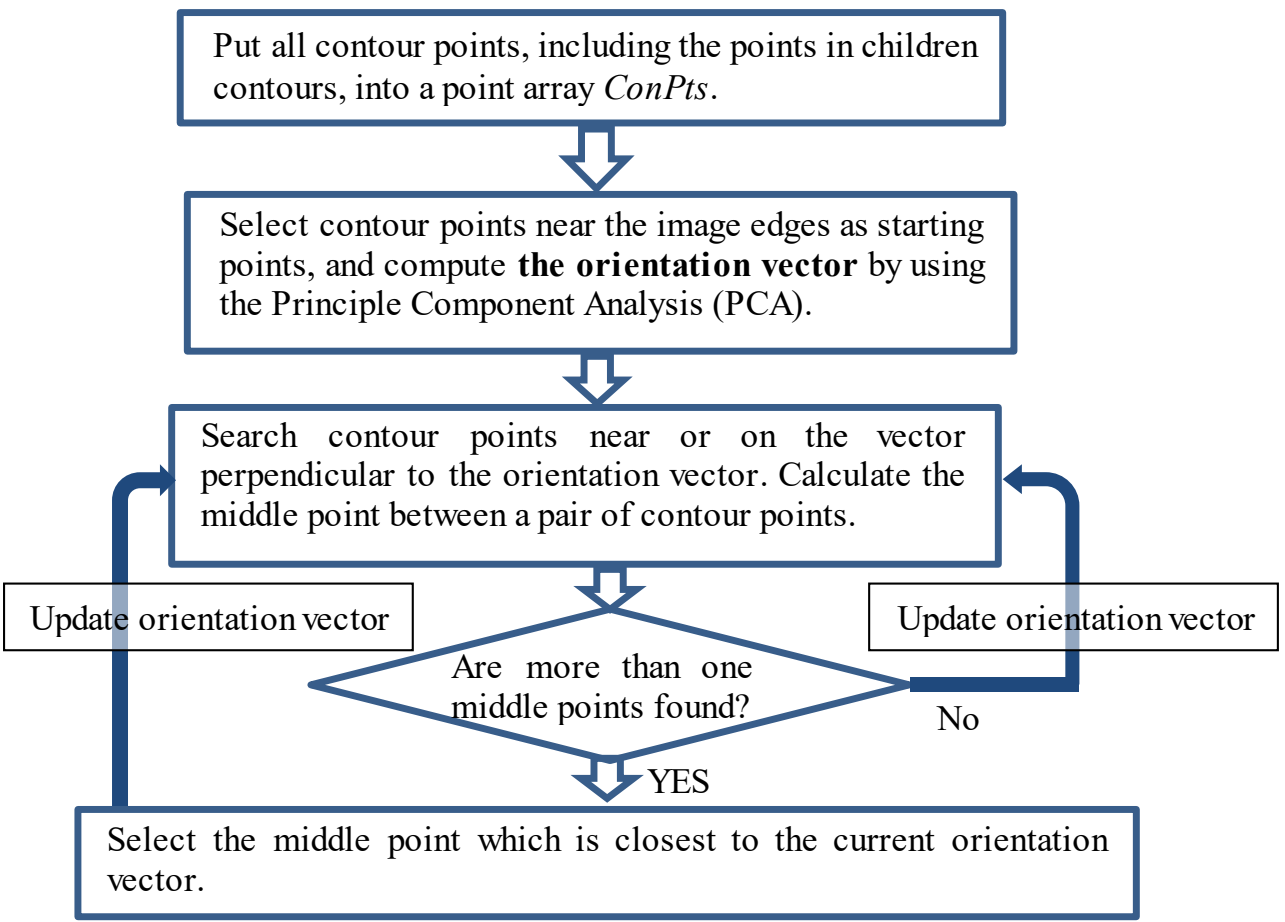


Fig. 5. Flow chart of finding the centerline from a contour block

In the hierarchy of contours, outer contours are called as parent and inner ones as child. If contours have children contours, it must have loops or intersections. In the final step of the algorithm, if the algorithm finds several middle points, the middle point which is closest to the current orientation vector will be selected. However, In the case of contour self-intersection, in order to increase the robustness, the algorithm will continue to find new middle points along the current orientation vector. The location of self-intersection can be detected by using children contours. The new orientation vectors are calculated from the new middle points. The middle points which create a sharp turning $(<90$ degree) between the current and new orientation vectors are removed. The final result is a smooth curve or line.

\subsection{Object Classification}

Although the vessel enhancement filter can enhance and detect wire-like objects, it could still be affected by some image artifacts, such as calcium deposits and rib bone boundaries (fig. 1). In order to recognize image artifacts, a k-nearest neighbour $(\mathrm{KNN})$ algorithm is used to separate target objects, such as guiding catheters and guidewire from image artifacts. Instead of applying KNN on the whole image to detect artifacts, a localized KNN image classifier is developed to process only wirelike objects, which are obtained from the previous object extraction step. First, 50 sample images of artifacts (negative data) and 50 sample images of target objects (positive data) are extracted from line blocks or contour blocks. They are manually labelled. The orientation vectors are computed and sample images are organized around the detected main axis. Then, the sample images were flipped along the $\mathrm{X}$ and $\mathrm{Y}$ axis to create a total of 200 sample images for both positive and negative data. This not only increases the number of training samples, but also solves the asymmetrical problem. The size of sample images is $20 \times 40$ pixels. Fig. 6 gives some examples. There are some bended wire images in positive data, which gives some flexibilities for KNN to recognize bended wires or catheters. All positive and negative sample images are normalized so that they all have the same value of the average intensity. This step is to prevent the wrong classification which is caused by the difference in the average intensity between two images. To normalize the image intensity, the image is subtracted by the intensity mean and then divided by the standard deviation of the intensity.

In current implementation, 5 nearest neighbours are used for KNN classifications. $\mathrm{KNN}$ uses Euclidean distances which are $d(p, q)=\sqrt{\sum_{i-1}^{N}\left(p_{i}-q_{i}\right)^{2}}$. Some of failed classifications are illustrated in figure 7 . 
(a) Positive data including guiding catheters and guidewires

(b) Negative data such as calcium deposit and rib bones

Fig. 6. Training samples for KNN (before the intensity normalization).

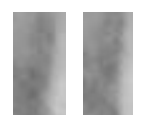

(a)

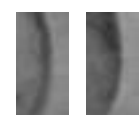

(b)

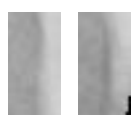

(c)

Fig. 7. Failed classifications for negative data (artifacts were classified as wire objects). (a) Intersection with a catheter. (b) X-ray contrast agent injection. (c) Calcium deposit.

As in the previous step, centerlines of contour blocks have been already computed, sub-images of $20 \times 40$ pixels can be extracted using centerlines and they will be normalized before feeding into the $\mathrm{KNN}$ image classifier. An example of classification is shown in fig. $9 \mathrm{~d}$ and $9 \mathrm{f}$.

\subsection{Wire Curve Reconstruction}

As there are many different ways to connect neighbour contour blocks, a minimum energy method is used to choose the smooth curve for the completed guiding catheter or guidewire. It could be defined as $E(s)=\int\left|d^{2} C(s) / d s^{2}\right|^{2} d s$, where $d^{2} C(s) / d s^{2}$ is the second order derivatives of catheter/guidewire curves. When the curve has $C^{1}$ continuity, it is a smooth curve. This mean that there are no sharp turning when connecting two contour blocks. Sharp turning is defined as $\left(\alpha_{i}+\alpha_{i+1}\right)>90^{\circ}$. A modified discrete energy function $E(s)=n+\sum_{i=0}^{n-1}\left(-\cos \alpha_{i}\right)$, where $\alpha_{i}$ is the connecting angle between the contour block and the connecting line (see fig. 8).

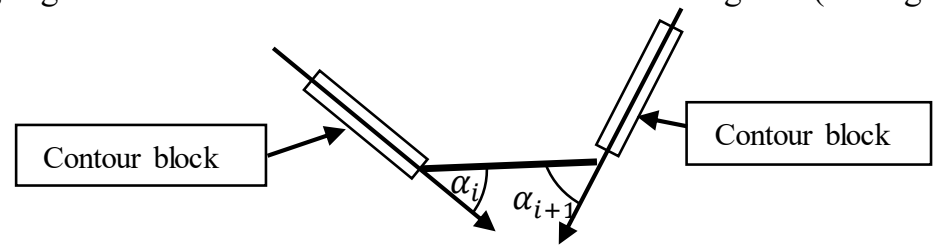

Fig. 8 connecting angles between 2 contour blocks

The distances between neighbour blocks should be also taken into consideration in the energy function. The final energy function is defined as $E(s)=n+$ $\sum_{i=0}^{n-1}\left(-\cos \alpha_{i}\right)+n * \frac{\sum_{i=0}^{n / 2-1} d_{i}}{L}$ where $d_{i}$ is the distance between two neighbour blocks 
and $L$ is the total length of reconstructed wire. The final part of the energy function is to minimize the distances between two neighbour blocks. The curve reconstruction algorithm uses a local optimization strategy as it is designed for real time processing. If the algorithm has to calculate all possible reconstruction paths (permutations), it will not be efficient for real-time processing. Also it will introduce delays in processing some images in the image sequence and will result in inconsistent frame rate of detection, as the number of found blocks varies from one image to another. Instead of calculating all permutations of all found blocks, we start with the longest blocks and search both ends for the next few blocks (in the current implementation, 3 blocks are used). After searching all possible paths, the one with minimum value of energy function is chosen.

\section{$3 \quad$ Results}

The localized machine learning method (KNN), was trained using 200 positive and negative sub-images, which are manually extracted from $25 \mathrm{X}$-ray images. They were acquired during 2 clinical cases. The proposed detection method was tested on 8 image sequences (1102 images), acquired in 3 different clinical cases. The frame size of each sequence is $512 \times 512$, with the pixel size between $0.368 \mathrm{~mm}$ and $0.433 \mathrm{~mm}$. To establish ground truth for evaluation, guiding catheters or guidewires are manually annotated by a clinical expert. An annotated object starts from the edge of the image and ends at its tip. They are used as the ground truth for accuracy tests. The overall detection precision is $0.87 \pm 0.53 \mathrm{~mm}$, which is defined as the average of shortest distances from points on a detected object to the corresponding annotated object. Furthermore, the shortest distances from the points in the annotated object to the detected object are calculated, which carries the penalty for not recovering the total length of the object in the ground truth. The result is $0.91 \pm 0.62 \mathrm{~mm}$, which is slight worse than the previous evaluation. The tip tracking precision is $0.54 \pm 0.36 \mathrm{~mm}$ for catheter tip and guidewire tip. The overall successful rate of detection is $83.4 \%$. For the technique to be acceptable in clinical practice, failed detections are considered to be the ones where any points on the detected object has larger errors than a preset threshold (e.g., a threshold of 4 image pixels [7] is used in this evaluation, which is the average radius of guidewires).

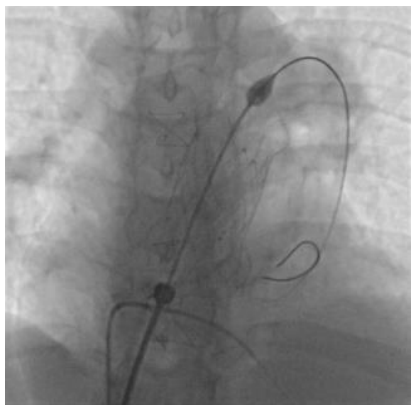

(a)

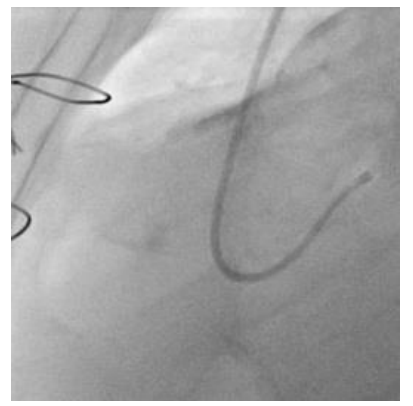

(c)

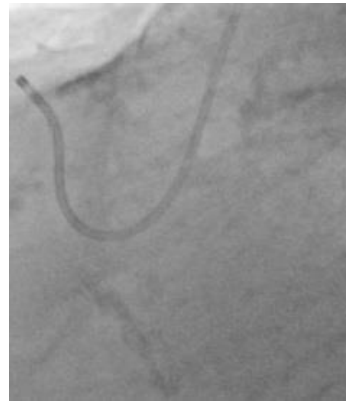

(e) 


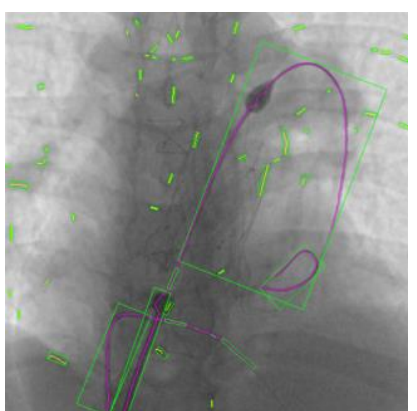

(b)

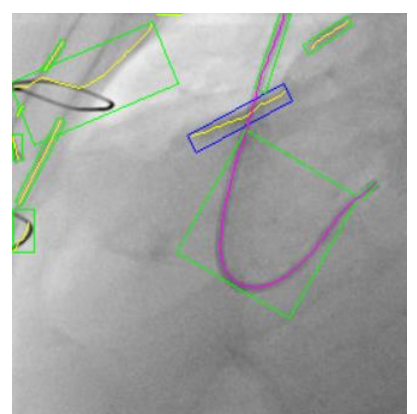

(d)

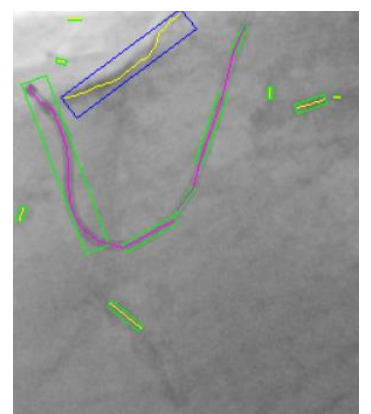

(f)

Fig. 9. (a)(c)(e) Origin X-ray images. (b)(d)(f) Purple lines are the detected guidewires. (d)(f) Blue rectangles are the contour blocks which are classified as image artifacts. The figures are best viewed in color.

The major computational load of the proposed method is the vessel enhancement filter algorithm. The software of the proposed method was implemented using OpenCV, and currently achieves a frame rate of $15 \mathrm{fps}$ using a single-threaded CPU implementation. The performance was evaluated on an Intel Core i7 2.7 GHz laptop.

\section{Conclusions and Discussions}

This paper describes a real-time guiding catheter and guidewire detection method. The proposed method does not require any user interaction or prior models. As the method is integrated with a localized machine learning algorithm, it can robustly distinguish between wire-like target objects and image artifacts. Therefore, the proposed method can efficiently and robustly work on low-dose X-ray images with a frame rate of $15 \mathrm{fps}$. This frame rate is considered as real-time for cardiovascular intervention procedures as the average maximum frame rate for modern intervention $\mathrm{X}$-ray systems is $15 \mathrm{fps}$. The detected models of guiding catheters or guidewires could be fed into a template-based tracking method, which can produce an even faster guidewire tracking method.

The proposed method is not limited to detect or track guiding catheters or guidewires. With some modifications of the localized machine learning algorithm, it can potentially detect intervention devices such as balloons, stents, and coils. Therefore, based on the proposed method, a general detection and tracking framework could be developed and used in CCI procedures, as well as other cardiac intervention procedures.

\section{Disclaimer}

Concepts/information are based on research and are not commercially available. 


\section{References}

1. Yilmaz A., Javed O., and Shah M., Object tracking: A survey. ACM Comput. Surv., 38(4):13, (2006).

2. Kass M., Witkin A., and Terzopoulos D., Snakes: Active contour models. International Journal of Computer Vision, 1(4), p321-331, (1987).

3. Zhu S. C. and Yuille A. L., Forms: A flexible object recognition and modeling system. International Journal of Computer Vision, 20:187-212, (1996).

4. D. Palti-Wasserman, A. M. Brukstein, and R. Beyar. Identifying and tracking a guide wire in the coronary arteries during angioplasty from x-ray images. IEEE Trans. Biomedical Engineering, 44(2), p152-164, (1997).

5. Baert S. A. M., Viergever M. A., and Niessen W. J., Guide wire tracking during endovascular interventions. IEEE Trans. Med. Imaging, 22(8), p965-972, (2003).

6. Barbu A., Athitsos V., Georgescu B., Boehm S., Durlak P., and Comaniciu D., Hierarchical learning of curves application to guidewire localization in fluoroscopy. In CVPR, (2007).

7. Wang P., Chen T., Zhu Y., Zhang W., Zhou S. K., and Comaniciu D., Robust guidewire tracking in fluoroscopy, in CVPR, (2009).

8. Pauly O., Heibel H., and Navab N., A machine learning approach for deformable guidewire tracking in fluoroscopic sequences, in MICCAI, (2010).

9. Frangi, A. F., Niessen, W. J., Vincken, K. L. and Viergever, M. A, Multiscale vessel enhancement filtering, in MICCAI, (1998).

10. Otsu N., A Threshold Selection Method from Gray-Level Histograms. IEEE Transactions on Systems, Man, and Cybernetics. 9(1), p62-66, (1979).

11. Fazlali H. R., et al, Vessel region detection in coronary X-ray angiograms in International Conference on Image Processing, (2015).

12. Y. L. Ma, N. Gogin, P. Cathier, R. J. Housden, G. Gijsbers, M. Cooklin, M. O'Neill, J. Gill, C. A. Rinaldi, R. Razavi and K. S. Rhode, Real-time x-ray fluoroscopy-based catheter detection and tracking for cardiac electrophysiology interventions, Medical Physics, 40(7), 071902-1 (2013).

13. E. Hecht, Optics. 4th ed., Addison Wesley, (2002). 